\title{
Move-it Sticky Notes Providing Active Physical Feedback through Motion
}

\author{
Kathrin Probst $^{1} \quad$ Michael Haller $^{1} \quad$ Kentaro Yasu $^{2} \quad$ Maki Sugimoto $^{3}$ Masahiko Inami $^{3}$ \\ ${ }^{1}$ Media Interaction Lab \\ University of Applied Sciences \\ Upper Austria \\ mi-lab@fh-hagenberg.at \\ ${ }^{2}$ Keio-NUS CUTE Center \\ National University \\ of Singapore \\ idmky@nus.edu.sg \\ ${ }^{3}$ Graduate School of Media Design \\ Keio University (KMD) \\ sugimoto@kmd.keio.ac.jp \\ inami@kmd.keio.ac.jp
}

\begin{abstract}
Post-it notes are a popular paper format that serves a multitude of purposes in our daily lives, as they provide excellent affordances for quick capturing of informal notes, and location-sensitive reminding. In this paper, we present Move-it, a system that combines Post-it notes with a technologically enhanced paperclip to demonstrate how a passive piece of paper can be turned into an "active" medium that conveys information through motion. We present two application examples that investigate the applicability of Move-it sticky notes for ambient information awareness. In comparison to existing notification systems, experimental results show that they reduce negative effects of interruptions on emotional state and performance, and provide unique affordances by combining advantages of physical and digital systems into a novel active paper interface.
\end{abstract}

\section{Author Keywords}

Active Paper Interface, SMA, Peripheral Display

\section{ACM Classification Keywords}

H5.2 [Information Interfaces and Presentation]: User Interfaces---Graphical User Interfaces, Interaction Styles

\section{General Terms \\ Design, Human Factors}

\section{INTRODUCTION}

In the course of everyday activities, people frequently take informal, personal notes that serve as temporary storage for information, or reminders for future action. Although many digital tools have been designed to support this ubiquitous behavior, traditional paper serves a function that, for many people, cannot be replicated on a screen. Therefore, paper still remains a frequently used medium that provides excellent affordances for fast and flexible capturing of information (e.g., jotting down notes during a phone call). It is likely readily at hand when needed, while also inexpensive, versatile, and plentiful in diverse forms [23].
Post-it notes, for example, are an iconic paper form factor that has many casual purposes in daily life, and serves as popular medium for informal note-taking [16]. Providing additional benefits of compactness and adhesiveness, they are frequently used for capturing information to be kept in mind (e.g., appointments, contacts, tasks), and stuck in prominent places as reminders. Their mere physical presence therefore provides excellent affordances for locationsensitive, passive reminding. Digital tools on the other hand, can provide active reminding functionalities, e.g., by presenting pop-up windows or alert sounds to notify of scheduled events, or incoming messages. Unfortunately, this makes them prominent sources for interruptions that are often perceived as annoying and frustrating, since they distract people from a primary activity, and may affect performance and emotional well-being negatively $[1,3]$.

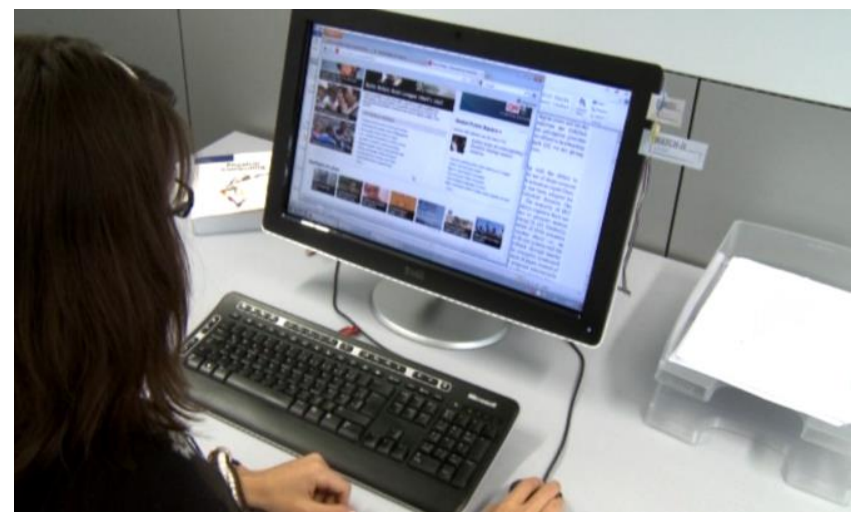

Figure 1: Move-it turns a passive piece of paper into an "active" medium.

In this paper, we present the design of an interface that combines Post-it notes (Move-it sticky notes) with a technologically enhanced paperclip (Move-it ioClip) to provide active feedback functionality (see Figure 1). Specifically, our contribution considers (1) the combination of traditional paper and digital information management to bridge the gap between the physical and digital world, (2) the addition of active motion feedback to turn a passive piece of paper into an "active" medium, (3) the utilization of subtle motion cues to optimize the tradeoff between attracting a user's attention and reducing negative effects of interruptions, and (4) the development of reusable hardware elements to augment traditional paper rather than modifying it. 
We explore the concept of providing active physical feedback with the design and evaluation of two application examples in the context of reminding (i.e., appointments) and information awareness (i.e., availability of remote contacts). Based on that, we discuss the advantages and limitations of the proposed active paper interface, and provide an overview of potential improvements and future directions.

\section{RELATED WORK}

We outline related work from three research areas relevant to this work: pen-and-paper interfaces that combine advantages of physical and digital interfaces, shape memory alloy technology that provides actuation capabilities to produce motion, and peripheral display research that aims to provide information awareness in the periphery of attention.

\section{Pen-and-Paper}

The fact that traditional paper is still very popular, albeit partially inferior to digital systems, has inspired researchers to investigate new ways of augmenting paper sketches with digital information $[9,14,24]$ or communication abilities $[2,18]$. Quickies, for example, use paper as I/O interface for handwritten notes, which are digitized and processed automatically [18]. In our setup, we aim to provide a similarly smooth transition between paper-based and digital notes through automatic integration with Personal Information Management (PIM) tools such as calendars, address books, or to-do lists. While feedback provided by most existing systems is of digital nature only, we extend this approach with physical feedback capabilities as similarly introduced by the Smart Filing System that highlights documents in a filing cabinet through LED lights [22]. In contrast to this work, however, we consider augmenting paper with motion feedback, which is particularly efficient in attracting human attention-especially in the peripheral vision [5].

\section{Shape Memory Alloy}

To provide traditional sticky notes with motion feedback capabilities, we make use of shape memory alloy (SMA), an active material that changes shape with respect to temperature. Given its light weight and flexibility, research has explored SMA as actuators for shape-changing interfaces $[7,19]$ or paper origami $[12,15,21]$. The Animated Paper Toolkit, for example, uses active materials to create animated paper toys [15]. In our work, we follow a similarly modular approach that integrates SMA into an everyday object, i.e., a paperclip, rather than embedding circuitry directly into paper. Thereby, we make use of a simple bending style actuation [15] to apply motion feedback for notifications as similarly explored with the MorePhone flexible smartphone prototype that changes shape to indicate incoming calls or messages [8]. In contrast to this work, however, we aim to explore actuated shape notifications with paper as base material, as it offers a number of appealing tangible characteristics (e.g., lightness, highly flexible deformation, look-and-feel of paper and ink).

\section{Peripheral Displays}

Taking advantage of the human ability to perceive even subtle changes in the periphery of attention, peripheral (or ambient) displays seek to support this kind of awareness to monitor information sources (e.g., news, weather, messages, remote presence) without distracting or disturbing a user engaged in a primary activity $[17,25]$. De Guzman et al., for example, introduce the design of peripheral displays in the context of instant messaging with various tangible interfaces embedded into everyday objects [10]. In our work, we apply a similar concept to move the information display off the screen, onto paper-based sticky notes that are directly integrated into the physical environment [13].

\section{MOVE-IT}

Figure 2 illustrates the typical lifecycle of a Move-it sticky note, which basically consists of the following steps:

1. Handwritten notes are captured on an augmented Post-it note (Move-it sticky note) using a digital pen. The strokes are transmitted to a desktop application, digitized and converted to text, and synchronized with a corresponding digital PIM tool (e.g., calendar).

2. The Move-it sticky note is attached to a prominent location (e.g., a monitor) for regular passive reminding.

3. The Move-it sticky note is combined with an intelligent paperclip (Move-it ioClip), which identifies the note by processing a unique pattern printed on the backside of the paper to establish a link between the physical paper note and its digital representation.

4. Upon occurrence of a specified event (e.g., a particular time, upon receiving a message or status update) feedback is provided by actuating the Move-it ioClip, hence bending the associated Move-it sticky note.

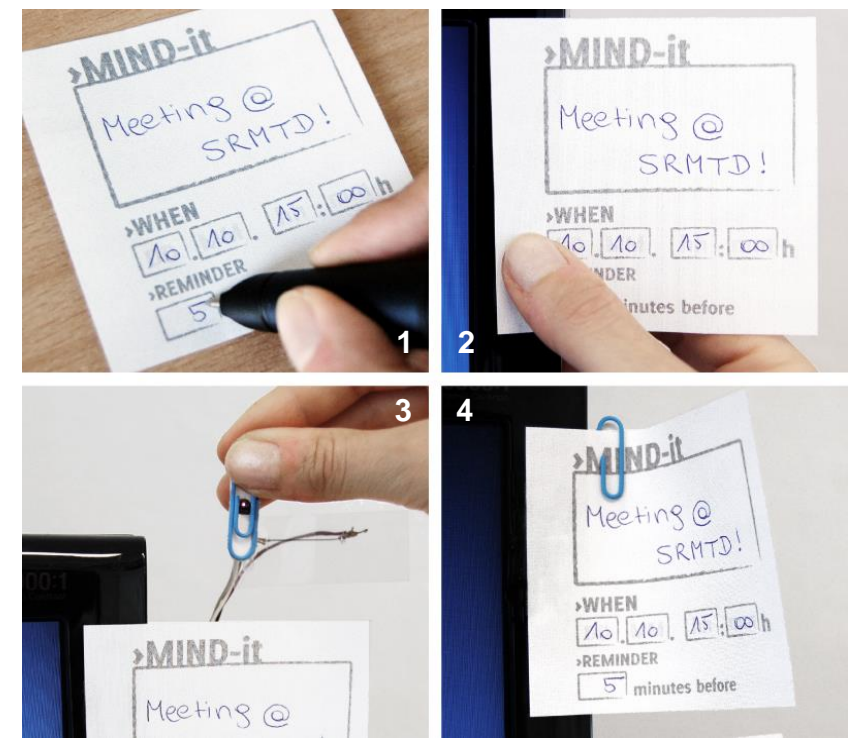

Figure 2: Handwritten notes are captured on paper (1), stuck to a prominent location (2), and combined with a technologically enhanced paperclip (3) that triggers motion feedback (4). 


\section{Tracking the Handwritten Notes}

With Move-it sticky notes, the note-taking process is as convenient and familiar as with traditional pen and paper. Notes are captured using a digital Anoto $^{1}$ pen that processes a unique dot pattern each Move-it sticky note is covered with. The digital pen transmits recorded strokes to the Move-it background application on the desktop, which converts the strokes to machine-readable text using the $\mathrm{Mi}$ crosoft Handwriting Recognition $S D K^{2}$. To reduce mental overhead for the user during the note-taking process [6], we use a form-based approach to classify the written strokes based on the input region they are written in. The result is then automatically synchronized with a digital PIM tool (i.e., Microsoft Outlook in our implementation). Thus, for example, an appointment reminder is visible on the physical sticky note as well as in the digital calendar.

\section{Identifying the Sticky Notes}

To determine which Move-it ioClip to actuate once a specified event occurs, it is necessary to establish a distinct association between the digital and physical notes. Therefore, we use reflective sensors that combine an infrared-emitting diode and phototransistor attached to the paperclip, which track the infrared light reflected from a unique grayscale pattern printed on the backside of each Move-it sticky note.

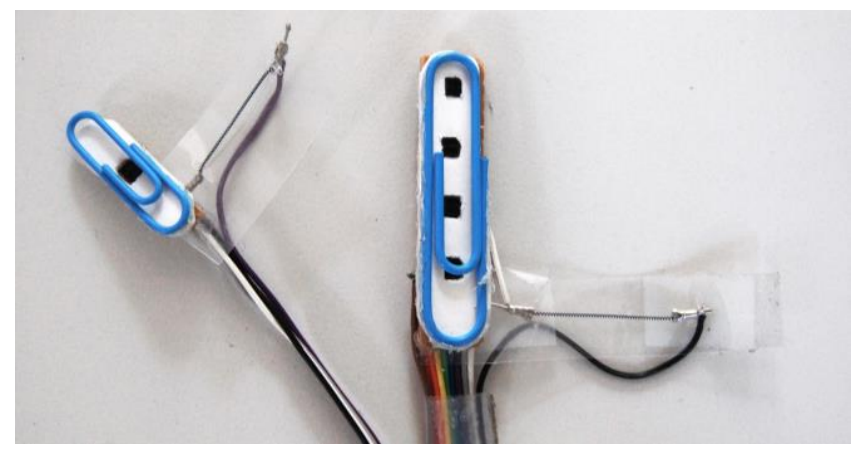

Figure 3: A Move-it mono-sensor (left) and multi-sensor (right) ioClip with reflective sensors for sticky note identification and SMA for actuation.

During the design process, we have developed two types of Move-it ioClips that are depicted in Figure 3. Mono-sensor ioClips use one reflective sensor to track a pattern consisting of single strip colored in a specific shade of gray. Thereby, we can reliably distinguish a maximum of ten different grayscales. Multi-sensor ioClips use four reflective sensors that track a pattern consisting of four strips, which are two strips in specific shades of gray that are enclosed by one black and one white strip (used for ambient light adjustment). While this design requires a different form factor (i.e., a larger size paperclip), it is less susceptible to ambient light interference, and provides robust tracking of up to $10 \times 10$ different patterns i.e., 100 Move-it sticky notes.

\footnotetext{
${ }^{1} \mathrm{http}: / /$ www.anoto.com

${ }^{2}$ http://msdn.microsoft.com/en-us/library/ms 754080
}

Reading the grayscale pattern on the backside of the paper enables each Move-it sticky note to be associated with a unique numeric identifier, created and managed by the Move-it desktop application. This ID is associated with the digital representation of the sticky note that serves as link between the Move-it ioClip and the Move-it sticky note.

\section{Actuating the Sticky Notes}

Once a specified event occurs, the Move-it sticky note is actuated by a Move-it ioClip to provide active physical motion feedback. Therefore, each paperclip is attached with a BMX $150^{3}$ spring (length $20 \mathrm{~mm}$; diameter $0.15 \mathrm{~mm}$ ) that is controlled by an Arduino Mega $2560^{4}$ microcontroller, and affixed to a small piece of polyester film. Once heated, the SMA shrinks to produce a bending actuation [15]. Thereby, the surface of the affixed polyester film is bended, which distorts the attached piece of paper (see Figure 4). Thus, combining a Post-it note with a Move-it ioClip, the paper can be actuated without direct embedding of circuitry.
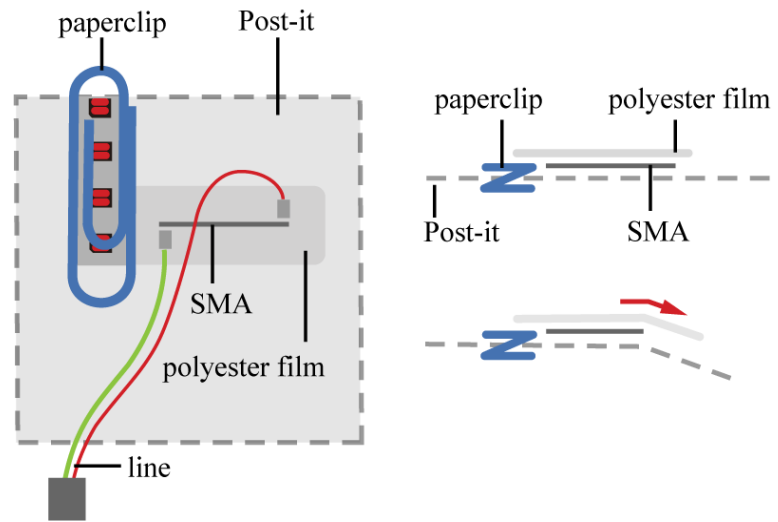

Figure 4: Schematic of a Move-it ioClip with attached SMA and polyester film (left) that shrink/bend when heated (right).

Once the SMA cools down, it returns to initial shape and unfolds the Post-it. Consequently, heating and cooling the SMA can produce arbitrary patterns of kinetic movement, which we use to generate two different actuation modes:

- In the first mode, the Post-it is bended repeatedly to create a wiggling motion. Periodic actuation impulses induce fast heating $(500 \mathrm{~ms})$ and shrinking of the SMA, followed by a cooling and relaxation period ( $2000 \mathrm{~ms})$. Actual motion duration may vary though, as actuation is considerably influenced by ambient temperature and material properties. In the final version, we achieved a maximum speed of approximately $0.3 \mathrm{~Hz}$, and bending range of $45^{\circ}$.

- In the second mode, the Post-it is bended once and retains shape for a longer period of time. For maintaining the bending angle, we apply pulse width modulation to avoid overheating. In our final implementation, we were able to keep a bending angle of $45^{\circ}$ for several hours.

\footnotetext{
${ }^{3}$ http://www.toki.co.jp

${ }^{4}$ http://arduino.cc/en/Main/ArduinoBoardMega2560
} 


\section{APPLICATION EXAMPLES}

To demonstrate the versatility of Move-it sticky notes, we implemented two application scenarios as proposed in our early work [20]: Mind-it sticky notes for active reminding, and Watch-it sticky notes for information awareness.

\section{Mind-it Sticky Notes for Active Reminding}

We are often confronted with large amounts of information to be remembered throughout our busy lives. Therefore, we make use of different kinds of reminders to highlight approaching events to be attended, or tasks to be completed [16]. This is well-supported by the affordances of paperbased reminders, such as lightweight capturing or enrichment with context information. The paper remains passive though, and the onus is on ourselves to become aware of the reminder in time. To address this problem, Mind-it sticky notes are designed to provide people with increased support for remembering notable information (e.g., events, tasks). Therefore, a Mind-it sticky note can for example remind us five minutes prior to a scheduled meeting through subtle wiggling motion (see Figure 5).

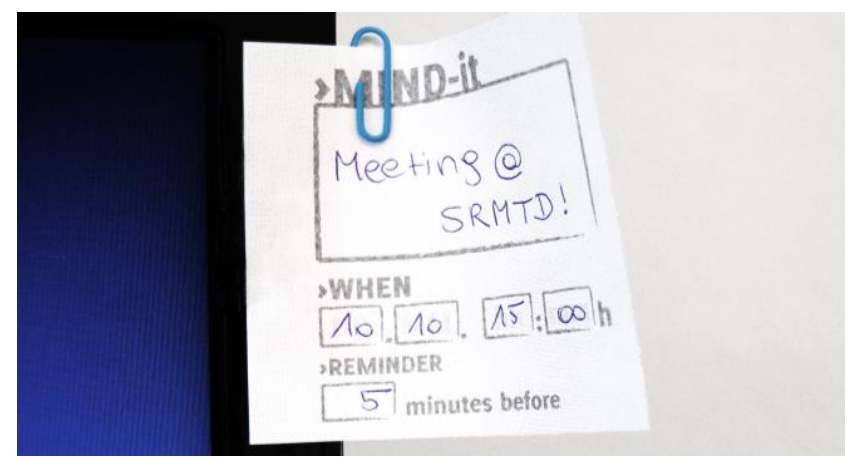

Figure 5: Once the deadline for an event approaches, a Mindit sticky note provides feedback through subtle motion.

Serving as location-based active reminders, Move-it sticky notes therefore provide affordances of paper-based and digital interfaces. As with traditional paper, notes are created conveniently through handwritten annotation, serve as passive reminders through their physical presence, and can be stuck in arbitrary places to provide location-based context. In addition, the notes are augmented with the ability to proactively attract attention through motion feedback.

\section{Watch-it Sticky Notes for Information Awareness}

In the course of a workday for example, we typically have many spontaneous conversations, and therefore often make use of digital communication tools (e.g., e-mail, instant messaging). Though, the utilization of such tools oftentimes requires considerable cognitive effort caused by constant monitoring activities, e.g., to maintain awareness of incoming messages, or presence of people in remote spaces. To address this problem, Watch-it sticky notes are designed to support people by visualizing the availability of remote contacts. Therefore, a Watch-it sticky note can for example keep us informed about the online status of our favorite IM contacts through shape changes (see Figure 6).

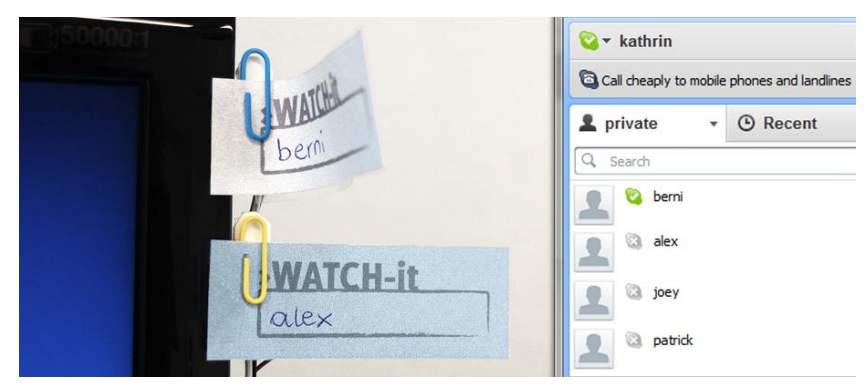

Figure 6: Once the availability of an IM contact changes, the shape of a Watch-it sticky note is adjusted accordingly.

Serving as peripheral information displays, Watch-it sticky notes therefore provide advantages over today's common digital tools such as filtering and grouping. As we usually may be interested in the availability of a few specific contacts, the sticky notes can be used to select the most important ones to be monitored. Further, the notes can be enriched with additional context information through grouping (e.g., spatial arrangement, color-coding). Status updates are indicated through smooth shape changes allowing us to determine the availability of an IM contact at a glance.

\section{EVALUATION}

Through two experiments, we explore the applicability of Move-it sticky notes for the proposed usage scenarios, and compare them to common solutions (i.e., dialog boxes, popup windows), which are currently widely adopted in commercially available PIM and communication tools.

\section{Feedback Modalities}

In the first experiment we compare Move-it sticky note feedback to standard Microsoft Outlook reminders, in the second experiment we compare Watch-it sticky note feedback to common Skype status updates. This results in two feedback modalities for each experiment:

- Move-it Sticky Note Feedback: In this condition, interruptions are communicated through wiggling Mind-it notes or shape-changing Watch-it sticky notes.

- Pop-Up Feedback: In this condition, interruptions are communicated through Microsoft Outlook calendar notifications or Skype status updates.

\section{Experimental Design}

18 participants ( 3 female; age $21-27$ ) were recruited from a local university. In both experiments, participants performed an attention-demanding primary task while simultaneously responding to interruptions presented at random intervals. Experiments were conducted on a desktop computer within a calm office environment. A custom application displayed task instructions, and recorded performance measures in the background. After each task, participants answered a modified NASA Task Load Index (TLX) survey [11] that contained additional scales related to potentially negative impacts of interruptions (i.e., disruption to the workflow, distraction by the feedback). 


\section{Experiment 1: Mind-it Sticky Notes for Alerting}

Reminding people of upcoming events is one of the typical application scenarios for Mind-it sticky notes, where they can serve as alerting displays that grab a user's attention as soon as important information appears [17]. To explore the applicability of Mind-it sticky notes, we compared them to Microsoft Outlook reminders, which are by default represented as dialog boxes in the center of the screen.

\section{Experimental Tasks}

During the experiment participants performed an attentiondemanding primary task, while periodically attending to a secondary task upon request. The primary task adapted from Adamczyk et al. [1] consisted in transcribing a news media clip. As secondary task, participants performed a short arithmetic training where they had to solve a sequence of ten basic calculations (e.g., $68+53$ ). Reminders were triggered at random intervals (100-120 seconds), requesting participants to attend to the secondary task. Simulating a realistic multitasking scenario, we instructed participants to focus on the primary task, and respond to the notifications (by pressing the 'ESC' key) within a reasonable timeframe. The tasks were designed to induce relatively high cognitive demand, as participants needed to deal with multiple different applications in parallel, and periodically memorize the text to be transcribed in the document.

Primary task performance was measured in terms of progress on the transcript, and number of errors in the text. Secondary task performance was measured in terms of time needed to solve the calculations, and number of errors.

\section{Procedure}

At the beginning of the experiment, participants partook in two short training sessions to become accustomed to the transcript and arithmetic tasks. Thereafter, the primary transcript task and secondary arithmetic task were combined, and participants performed them in the sticky-note and popup conditions in counter-balanced order, with three interruptions per condition (see Figure 7). After each condition, participants answered a NASA TLX questionnaire.

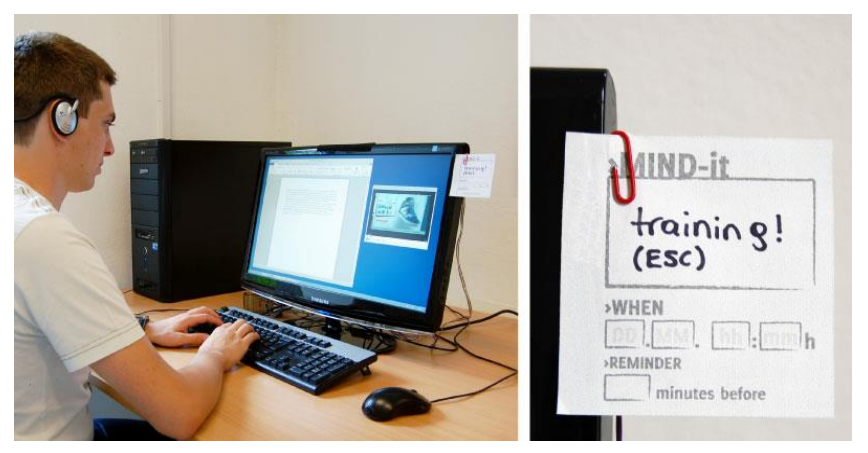

Figure 7: Apparatus of the first experiment (left), with a Mind-it sticky note attached to a computer monitor (right).
Results

Statistical analysis using within-subjects ANOVA showed no main effect on task performance in the primary or secondary task. Analyzing the times needed to start the arithmetic training, however, we found that participants took significantly longer in the sticky note condition $(M=11.28$ $\mathrm{s}, S D=3.95)$ than the pop-up condition $(M=7.40 \mathrm{~s}, S D=$ $3.43), F_{1,17}=7.30, p<.05$. These results are supported by further in-depth analysis, which revealed that participants' tendency to postpone the secondary task to a certain degree (e.g., finish typing a word or sentence before leaving the primary task) was significantly higher in the sticky note condition $(M=81.48 \%, S D=20.52)$ than the pop-up condition $(M=37.04 \%, S D=22.55)$. While this may be largely attributed to the fact that digital reminders were presented on-screen, whereas sticky notes provided feedback in the periphery, previous research on information awareness suggests that such postponed awareness strategies are likely associated with a perceived lower level of intrusiveness [4].

Further, analyzing the NASA-TLX ratings (see Figure 8) a Wilcoxon Signed Rank test showed a main effect of feedback modality on participants' task load ratings for performance $(Z=2.683, p<.01)$ and effort $(Z=2.392, p<.05)$ in favor of the sticky notes. Moreover, ratings indicate that notifications announcing the secondary task were perceived less negatively in the sticky note condition, according to the custom scales for workflow $(Z=3.524, p<.01)$ and distraction $(Z=3.523, p<.01)$. There was no effect on the remaining workload-related scales.
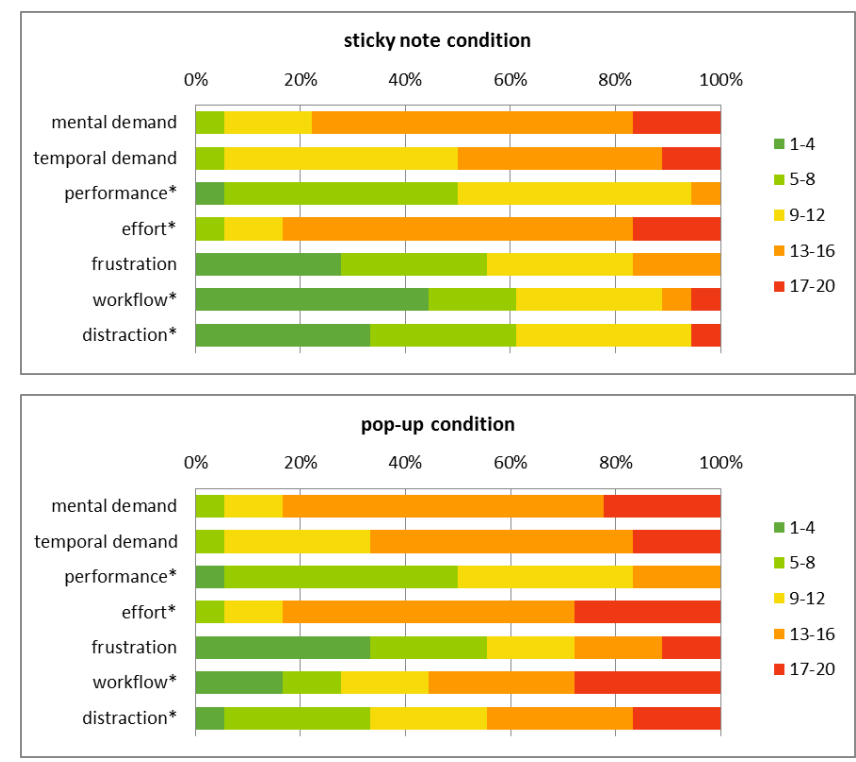

Figure 8: NASA-TLX ratings of the first experiment for sticky note and pup-up feedback (*indicates statistical significance). 


\section{Discussion}

Summarizing, the results of the first experiment indicate that participants perceived Mind-it sticky notes significantly less disrupting than digital Microsoft Outlook reminders. Within the experiment most participants responded to the feedback immediately by switching to the arithmetic training task, which was also confirmed in participants' comments, e.g., "When I was informed to perform the arithmetic training by the Post-it notes, I could ignore the feedback for one moment to finish my current line of thought. The Outlook reminders were particularly disturbing, because I was completely distracted from my current activity". This suggests that participants were less stressed by the sticky note feedback, hence perceived the interruptions less disruptive and annoying. However, although participants perceived to accomplish the task more successfully in the sticky note condition, performance measurements did not confirm these perceptions. This might be due to the fact that tasks in the experiment were relatively tolerant to interruptions (typing speed and errors were not affected fundamentally).

Moreover, while we used Microsoft Outlook as reference in this experiment, it is noteworthy to say that representation of reminders as dialog boxes is in fact state-of-the-art behavior, as applied by most desktop-based notification systems to attract users' attention effectively. In this context, some participants commented that feedback provided by Move-it sticky notes could be too subtle in real-world situations. This could be explored in an in-situ field deployment of Move-it sticky notes. Another interesting insight during the study was provided by eight participants (i.e., $44 \%$ ) commenting on recognizing subtle auditory cues provided by the sticky notes, which were generated by the polyester film scrubbing along back of the paper note when actuated. However, none of them indicated to perceive this effect negatively, but rather as notable additional feedback cue.

\section{Experiment 2: Watch-it Sticky Notes for Awareness}

Supporting awareness of the presence of remote people is one of the typical application scenarios for Watch-it sticky notes, where they can serve as ambient displays that remain in the periphery [17]. To explore the applicability of Watchit sticky notes for awareness support, we compared them to Skype status updates, which are by default represented as pop-up windows in the lower right corner of the screen.

\section{Experimental Tasks}

During the experiment, participants performed an attentiondemanding primary task, while maintaining awareness of an information source in the periphery. The primary task consisted in an object-catching game, where continuously generated falling shapes needed to be caught by controlling the horizontal position of a virtual bat with the mouse. As peripheral task, participants had to monitor the availability status (i.e., online, offline) of three fictional instant messenger contacts.
Status updates were triggered at random intervals (10-20 seconds), which participants had to acknowledge by pressing a corresponding key on the keyboard (i.e., first letter of the contact name). Simulating a realistic gaming scenario, we instructed participants to focus on the primary task, and acknowledge status updates within a reasonable timeframe. The tasks for this experiment were designed to be relatively attention-demanding and error-critical, with high density (generation interval $=750 \mathrm{~ms}$ ) and speed (fall duration 2-3 seconds) assigned to falling objects in the gaming task.

Primary task performance was measured in terms of number of shapes caught during the game. Secondary task performance was measured in terms of time needed to acknowledge the status update (awareness latency) and number of correct acknowledgments (awareness achievement).

\section{Procedure}

At the beginning of the experiment, participants completed two short training sessions to become accustomed to the gaming and status monitoring tasks. The keyboard assignment was announced prior to the training, and remained the same for the rest of the experiment. Thereafter, the primary gaming task and peripheral status monitoring task were combined, and participants performed them in the sticky note and pop-up conditions in counter-balanced order with twelve interruptions each (see Figure 9). After each condition, participants answered a NASA TLX questionnaire.

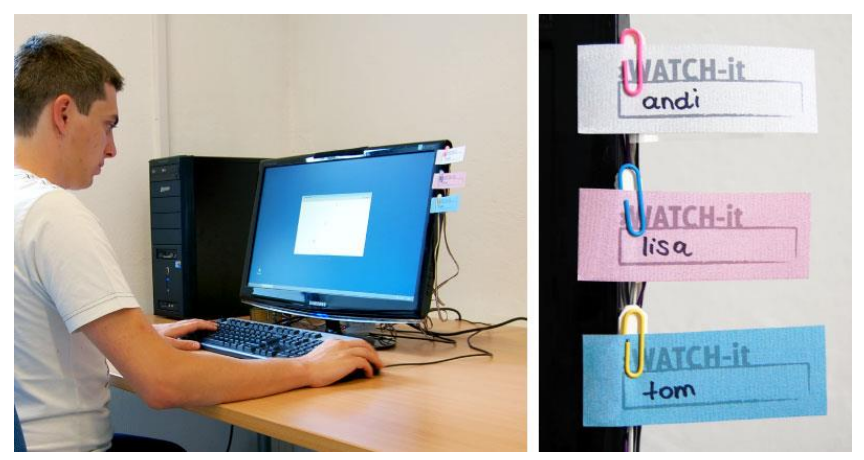

Figure 9: Apparatus of the second experiment (left), with three Watch-it sticky notes attached to a computer monitor (right).

\section{Results}

Statistical analysis of the primary task performance revealed a main effect of feedback modality on success rate i.e., the number of objects caught in the gaming task $\left(F_{1,17}=\right.$ $15.11, p<.01)$, with participants achieving an average success rate of $97.23 \%(S D=1.66)$ in the sticky note condition, and $95.59 \%(S D=2.10)$ in the pop-up condition. Furthermore, there was an effect on awareness latency $\left(\mathrm{F}_{1,17}=\right.$ 43.28, $p<.01)$, with response times being significantly higher in the sticky note condition $(M=2.13 \mathrm{~s}, S D=0.31)$ than the pop-up condition $(M=1.72 \mathrm{~s}, S D=0.19)$. Awareness achievement was close to $100 \%$ in both conditions and did not show a main effect. 
Ratings of the NASA-TLX survey largely confirm the findings of the first experiment. The tasks were perceived to be accomplished with better performance $(Z=2.272, p<.05)$ and less effort $(Z=2.139, p<.05)$ in the sticky condition. Moreover, contact status updates had more positive ratings on the custom scales for workflow $(Z=2.421, p<.05)$ and distraction $(Z=3.523, p<.01)$ in the sticky note condition.

\section{Discussion}

Summarizing, the results of this experiment are consistent with the findings of the first experiment regarding the less disturbing effect of the actuated paper notes. As the gaming task in the present experiment was more error-critical, the results of the task load ratings were reinforced through increased task performance in the sticky note condition, with significantly higher success rates when feedback was provided by Watch-it sticky notes.

Although Skype notifications are less intrusive than Microsoft Outlook reminders (located in the lower right corner of the screen, no immediate response required), results of the experiment indicate that Watch-it sticky notes were perceived even less disruptive. Partially, this might be due to the fact that Skype notifications required reading and processing the contact name for each status update, whereas Mind-it sticky notes were constantly present at the side of the monitor so that participants could familiarize with their spatial arrangement and colors. From participants' comments we noticed a number of statements confirming these results, e.g., "After a while I could easily determine from my peripheral vision which contact's status had changed", "Since each Post-it note was assigned a specific position and color, there was no need to re-read the contact name every time". Increased response times in the sticky note condition may be largely attributed to the fact that the paper notes allowed for processing without shifting focus of attention off the primary task. Thus, to a certain degree, participants could time acknowledgements to opportune moments in the course of the game-a phenomenon that was observed with twelve participants (i.e., $66 \%$ ).

\section{ADVANTAGES AND LIMITATIONS}

From the experimental results and participants' comments, we can summarize that the different types of Move-it sticky notes provide a number of unique affordances:

- Lightweight note capturing through familiar handwritten annotation using pen and paper.

- Positioning in arbitrary places to provide permanent visibility, and location-based context information.

- Support for enrichment with context information through spatial arrangement or color-coding.

- Digital processing of handwritten input and automatic integration with digital PIM tools.

- The ability to provide active motion feedback and attract users' attention once associated with a Move-it ioClip.
During the design process of Move-it sticky notes, one of our main considerations was to preserve the fundamental affordance of paper being a light, cheap, and disposable material. However, while the combination of a common paperclip with SMA is relatively simple from a design perspective, the technology comes with limitations like heating problems, speed limitations due to dependency on environmental parameters (e.g., temperature), and need of wiring to provide power supply for actuation [15,21]. Potential solutions addressing these issues will be investigated in our future work include the use of Peltier elements for controlling SMA temperature, selective inductive powering to reduce external wiring [26], and exploration of ferromagnetic SMA that changes shape in response to magnetic fields.

Although Anoto technology and similar solutions are nowadays more and more present in modern office environments, flexibility and portability of Move-it is currently also limited by the need for specially printed paper, and connection to a computer for synchronization of handwritten notes. However, as this is only one possible implementation of analog-to-digital note capturing, the currently used technology could easily be replaced by other technologies (e.g., ultrasonic tracking, pens with internal storage).

Other limitations remaining in the current version of our implementation include limited support for posterior correction or modification of handwritten annotations, or constraints of the form-based layout approach. Free-form annotation might therefore be more attractive in terms of input flexibility, but raises specific requirements regarding automatic content recognition (e.g., content types/formats, clustering) to be solved. Apart from that, we think that particularly for note-taking scenarios, paper-based sticky notes provide a number of appealing characteristics and material properties that are currently not yet equally replicated with digital equivalents like flexible e-paper displays (e.g., lightness, highly flexible deformation, fast and easy annotation). Creating e-paper versions of Move-it sticky notes will become feasible as soon as digital paper is broadly available and inexpensive, and users can write on it fast and freely. This would also decrease the amount of paper consumed with traditional Post-it notes.

\section{CONCLUSION AND FUTURE WORK}

In this paper, we presented Move-it sticky notes that combine advantages of physical and digital note-taking, and provide additional physical feedback capabilities through subtle motion cues. We explored their application for two specific computing scenarios (i.e., reminding, information awareness), and reported the results of two experiment that show significantly less disruptive effects for Move-it sticky notes in comparison to widely adopted state-of-the-art digital notifications. User feedback indicates that the unique affordances of the proposed active paper interface are perceived as supportive, and the concept of providing active motion feedback capabilities is a potentially helpful extension to traditional paper. 
In our future work, we aim to reduce the amount of wiring that is currently limiting portability of the paper notes, and investigate how scaling the approach by using a large number Move-it sticky notes influences user behavior. Furthermore, we plan to investigate additional ways of augmenting Move-it sticky notes with the ability to sense user input through physical manipulation (e.g., folding, tearing, crumpling). A sticky note could for instance react to a dog-eared corner by opening the digital item in the PIM tool on the desktop computer, while tearing or crumpling could invoke the deletion of the associated digital representation. Finally, we plan to explore the use of Move-it sticky notes in a realworld setting, e.g., an office environment.

\section{ACKNOWLEDGEMENTS}

This research is supported by the Austrian Research Promotion Agency GmbH (FFG) within the Bridge project \#2579917 and the Singapore National Research Foundation under its International Research Center Keio-NUS CUTE Center @ Singapore Funding Initiative and administered by the IDM Program Office.

\section{REFERENCES}

1. Adamczyk, P. and Bailey, B. If Not Now, When?: The Effects of Interruption at Different Moments Within Task Execution. Proc. CHI 2004, ACM, 271-278.

2. Arregui, D., Fernstrom, C., Pacull, F., et al. Paperbased Communicating Objects in the Future Office. Proc. SOC 2003.

3. Bailey, B., Konstan, J., and Carlis, J. The Effects of Interruptions on Task Performance, Annoyance, and Anxiety in the User Interface. Proc. Interact 2000, IOS Press, 593-601.

4. Bailey, B., Konstan, J., and Carlis, J. Adjusting Windows: Balancing Information Awareness with Intrusion. Proc. HFWeb 2000.

5. Bartram, L., Ware, C., and Calvert, T. Moving Icons: Detection And Distraction. Proc. Interact 2001, IOS Press, 157-165.

6. Bernstein, M., Van Kleek, M., Karger, D., and schraefel, m.c. Information Scraps: How and Why Information Eludes our Personal Information Management Tools. ACM TOIS 26, 4 (2008).

7. Coelho, M. and Zigelbaum, J. Shape-Changing Interfaces. Personal and Ubiquitous Computing 15, 2 (2011), 161-173.

8. Gomes, A. and Nesbitt, A. MorePhone: A Study of Actuated Shape Deformations for Flexible Thin-Film Smartphone Notifications. Proc. CHI 2013, 583-592.

9. Guimbretière, F. and Park, C. Paper Augmented Digital Documents. Proc. UIST 2003, ACM, 51-60.

10. De Guzman, E., Yau, M., Gagliano, A., Park, A., and Dey, A. Exploring the Design and Use of Peripheral
Displays of Awareness Information. Ext. Abstracts CHI 2004, ACM, 1247-1250.

11. Hart, S. and Staveland, L. Development of NASA-TLX (Task Load Index): Results of Empirical and Theoretical Research. In Human Mental Workload. North Holland Press, 1988, 139-183.

12. Hawkes, E., An, B., Benbernou, N., et al. Programmable Matter by Folding. PNAS 107, 28 (2010).

13. Ishii, H. and Ullmer, B. Tangible Bits: Towards Seamless Interfaces between People, Bits and Atoms. Proc. CHI 1997, ACM, 234-241.

14. Klemmer, S., Newman, M., Farrell, R., Bilezikjian, M., and Landay, J. The Designers' Outpost: A Tangible Interface for Collaborative Web Site Design. Proc. UIST 2001, ACM, 1-10.

15. Koizumi, N., Yasu, K., Liu, A., Sugimoto, M., and Inami, M. Animated Paper: A Toolkit for Building Moving Toys. ACM CIE 8, 2 (2010), 7.

16. Lin, M., Lutters, W., and Kim, T. Understanding the Micronote Lifecycle: Improving Mobile Support for Informal Note Taking. Proc. CHI 2004, ACM, 687-694.

17. Matthews, T., Dey, A., Mankoff, J., Carter, S., and Rattenbury, T. A Toolkit for Managing User Attention in Peripheral Displays. Proc. UIST 2004, ACM, 247-256.

18. Mistry, P. and Maes, P. Augmenting Sticky Notes as an I/O Interface. Proc. UAHCI 2009, Springer, 447-556.

19. Poupyrev, I., Nashida, T., Maruyama, S., Rekimoto, J., and Yamaji, Y. Lumen: Interactive Visual and Shape Display for Calm Computing. Proc. SIGGRAPH 2004, ACM, 17.

20. Probst, K., Seifried, T., Haller, M., Sugimoto, M., Yasu, K., and Inami, M. Move-It: Interactive Sticky Notes Actuated by Shape Memory Alloys. Ext. Abstracts CHI 2011, ACM, 1393-1398.

21. Qi, J. and Buechley, L. Animating Paper using Shape Memory Alloys. Proc. CHI 2012, ACM, 749-752.

22. Seifried, T., Jervis, M., Haller, M., Masoodian, M., and Villar, N. Integration of Virtual and Real Document Organization. Proc. TEI 2008, ACM, 81-88.

23. Sellen, A. and Harper, R. The Myth of the Paperless Office. MIT Press, 2003.

24. Steimle, J. Designing Pen-and-Paper User Interfaces for Interaction with Documents. Proc. TEI 2009, ACM, 197-204.

25. Wisneski, C., Ishii, H., Dahley, A., et al. Ambient Displays: Turning Architectural Space into an Interface between People and Digital Information. Proc. CoBuild 1998, Springer, 22-32.

26. Zhu, K., Newton, O., and Cheok, A. Selective Inductive Powering System for Paper Computing Categories and Subject Descriptors. Proc. ACE 2011, ACM, 59. 